\title{
Atrial fibrillation following treatment with paclitaxel: A case report
}

\author{
DEHUA ZHAO ${ }^{1}$, JING CHEN ${ }^{1}$, XIAOJUN LIU ${ }^{2}$, XIAOQING LONG ${ }^{2}$, LISHA CAO ${ }^{1}$ and JISHENG WANG ${ }^{1}$ \\ Departments of ${ }^{1}$ Clinical Pharmacy and ${ }^{2}$ Oncology, The Third Hospital of Mianyang \\ (Sichuan Mental Health Center), Mianyang, Sichuan 621000, P.R. China
}

Received April 16, 2018; Accepted October 10, 2018

DOI: $10.3892 /$ br.2018.1158

\begin{abstract}
Paclitaxel (PTX) is an antimicrotubule agent, and is effective in treating a wide range of solid tumors. However, its use may lead to cardiovascular toxicities, the manifestations of which include arrhythmia, heart failure, acute myocardial ischemia and atrial fibrillation (AF). AF is among the severe reactions to the PTX cardiotoxicity, and a cause for substantial morbidity and mortality. However, the incidence of PTX-induced AF is reportedly low (1.0-1.7\% worldwide), and few cases have been reported in the literature. Thus, to emphasize the need for awareness of this side effect of PTX among clinicians, the report herein presents a case of AF induced by PTX in a patient with non-small-cell carcinoma. A 51-year-old man experienced AF following treatment with PTX. Amiodarone and metoprolol were administered to the patient to control cardiac rhythm and rate. After 3 days, the electrocardiogram was normalized and indicated normal heart rate and rhythm. According to this case, thorough attention should be paid during PTX treatment to monitor for signs of AF or other abnormalities in cardiac function.
\end{abstract}

\section{Introduction}

Paclitaxel (PTX) is commonly used for the treatment of various malignancies, including breast, lung, ovarian and other cancers (1). The major adverse reactions of this drug include alopecia, bone marrow suppression, polyneuropathy and cardiovascular toxicities (2). The incidence of cardiovascular toxicities in patients receiving PTX is $12-13 \%$ worldwide (3). Manifestations of the cardiovascular toxicities include atrial arrhythmias, asymptomatic bradycardia, left bundle branch block, ventricular tachycardia, congestive cardiac failure and atrial fibrillation (AF) $(3,4)$. AF is among the most critical adverse effects, though is relatively rare with an incidence

Correspondence to: Dr Jisheng Wang, Department of Clinical Pharmacy, The Third Hospital of Mianyang (Sichuan Mental Health Center), 190 Jiannan East Street, Mianyang, Sichuan 621000, P.R. China E-mail: 519425004@qq.com

Key words: paclitaxel, cardiovascular toxicities, atrial fibrillation, non-small-cell carcinoma rate of 1.0-1.7\% worldwide (5). The main mechanisms underlying PTX-induced AF are considered to be adrenergic or vagal stimulation, changing atrial conduction, refractoriness, automaticity, coronary vasoconstriction or ischemia, local electrolyte disturbances, and direct cardiotoxicity (5).

According to the literature, PTX may cause AF, particularly in elderly or patients with a history of cardiovascular disease, but also in patients with no cardiac risk factors $(5,6)$. Therefore, the possibility of AF should be considered in patients who develop arrhythmia or other symptoms following receipt of PTX. This is indicated in the present report, which presents a case of AF induced by PTX in a patient with non-small-cell carcinoma.

\section{Case report}

A 51-year-old Chinese male ex-smoker with stage IIIB (T4N2M0) non-small-cell carcinoma (7) presenting with right hilar and carina lymph node metastasis, diagnosed on August 2, 2016 at the Third Hospital of Mianyang (Mianyang, China). The patient had no history of diabetes, hypertension or cardiac illness, and his base line electrocardiogram (ECG) was normal (Fig. 1). The patient's heart rate was 82 beats per minute (bpm), and the QRS duration, and QT and PR intervals were 80,384 and $151 \mathrm{msec}$, respectively. He started the first cycle of combination chemotherapy with PTX and cisplatin (TP; PTX, $135 \mathrm{mg} / \mathrm{m}^{2}$ on day 1 and cisplatin, $25 \mathrm{mg} / \mathrm{m}^{2}$ on days 1-3) on September 30, 2016.

Three weeks later, the patient underwent the second cycle of chemotherapy with TP. Dexamethasone (20 mg per os) was administered $\sim 12$ and $6 \mathrm{~h}$ before PTX, and diphenhydramine [50 mg intravenous (iv)] and cimetidine [300 mg (iv)] were administered 30-60 min prior to PTX. At 2 days after administration of PTX, the patient's heart rate increased to $160 \mathrm{bpm}$ (normal range 60-90 bpm), which was accompanied by mild dizziness and shortness of breath, but with no obvious heart palpitations. The ECG indicated a rapid AF with rapid ventricular rate (Fig. 2). A diagnosis of AF was made. The patient was immediately administered amiodarone (150 mg bolus then $300 \mathrm{mg}$ continuous infusion). Two hours later, the symptom of shortness of breath had disappeared, and the heart rate had decreased to $106 \mathrm{bpm}$. Subsequently, metoprolol was administered to the patient to reduce heart rate, and three days later, the ECG was normalized and indicated normal heart rate and rhythm (Fig. 3). 
Table I. Naranjio algorithm score assignment.

\begin{tabular}{lccc} 
Question & \multicolumn{2}{c}{$\begin{array}{c}\text { No or Do } \\
\text { not know }\end{array}$ Score } \\
\hline 1. Are there previous conclusive reports on this reaction? & Yes & +1 & +1 \\
2. Did the adverse events appear after the suspected drug was given? & +2 & -1 & +2 \\
3. Did the adverse reaction improve when the drug was discontinued? & +1 & 0 & 0 \\
Or when a specific antagonist was given? & & & \\
4. Did the adverse reaction appear when the drug was readministered? & +2 & -1 & 0 \\
5. Are there alternative causes that could have caused the reaction? & -1 & +2 & +2 \\
6. Did the reaction reappear when a placebo was given? & -1 & +1 & 0 \\
7. Was the drug detected in any body fluid in toxic concentrations? & +1 & 0 & 0 \\
8. Was the reaction more severe when the dose was increased, & +1 & 0 & 0 \\
or less severe when the dose was decreased? & & & \\
9. Did the patient have a similar reaction to the same or similar drugs in any previous exposure? & +1 & 0 & 0 \\
10. Was the adverse event confirmed by any objective evidence? & +1 & 0 & +1 \\
Total & - & - & 6
\end{tabular}

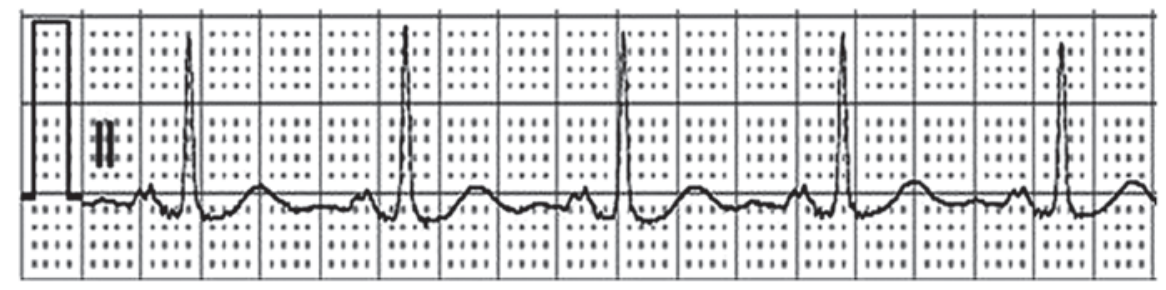

Figure 1. Electrocardiogram prior to adjuvant chemotherapy with paclitaxel and cisplatin.

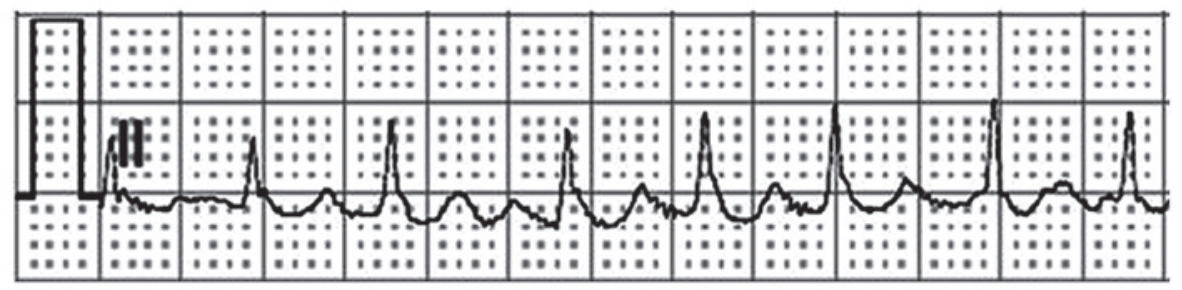

Figure 2. Electrocardiogram following adjuvant chemotherapy with paclitaxel and cisplatin.

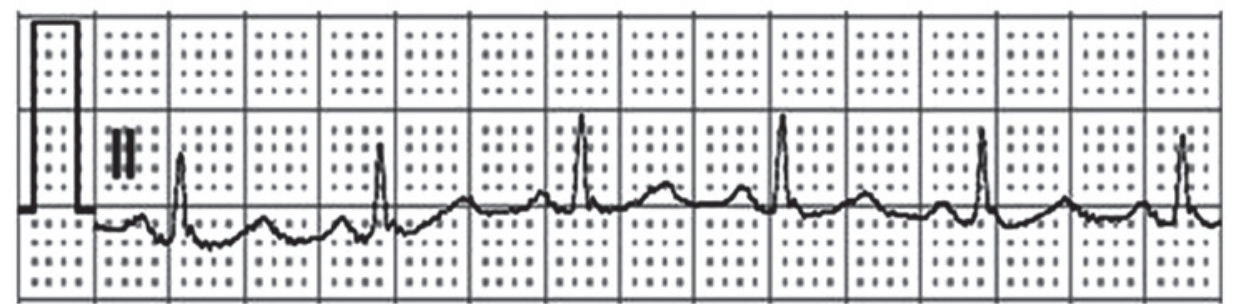

Figure 3. Electrocardiogram following administration of amiodarone and metoprolol.

To confirm the association between PTX and AF in the present case, the Naranjio algorithm $(8,9)$ was used to evaluate the potential causal relationship between PTX and AF. According to the Naranjio algorithm, the score was 6 points (Table I), indicating that the occurrence of AF was likely to be associated with use of PTX. Scoring was based on the following: i) There have been previous conclusive reports on this reaction (10-12), and therefore a score of 1 point was given; ii) 2 days after administration of PTX, the patient developed AF, and therefore 2 points were given; iii) in 
Table II. Hematological and biochemical parameters prior to and following chemotherapy.

Measured values

Auxiliary examination

Pre-chemotherapy Post-chemotherapy

Normal range

Routine

Neutrophils $\left(10^{9} / 1\right)$

Hemoglobin $(\mathrm{g} / \mathrm{l})$

Platelets $\left(10^{9} / 1\right)$

Hepatic function

Alanine aminotransferase (U/l)

Aspartate aminotransferase (U/l)

Total bilirubin $(\mu \mathrm{mol} / \mathrm{l})$

Renal function

Creatinine clearance $(\mathrm{ml} / \mathrm{min})$

Blood urea nitrogen ( $\mathrm{mmol} / \mathrm{l})$

Cardiac function

N-terminal pro-B-type natriuretic peptide $(\mathrm{pg} / \mathrm{ml})$

Creatine kinase MB (U/l)

Cardiac troponin I $(\mu \mathrm{g} / \mathrm{ml})$
5.8

97

239

0.01
5.2

91

214

$120-160$

100-300

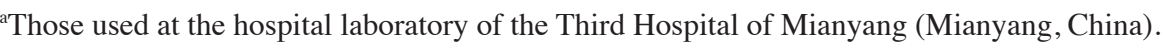

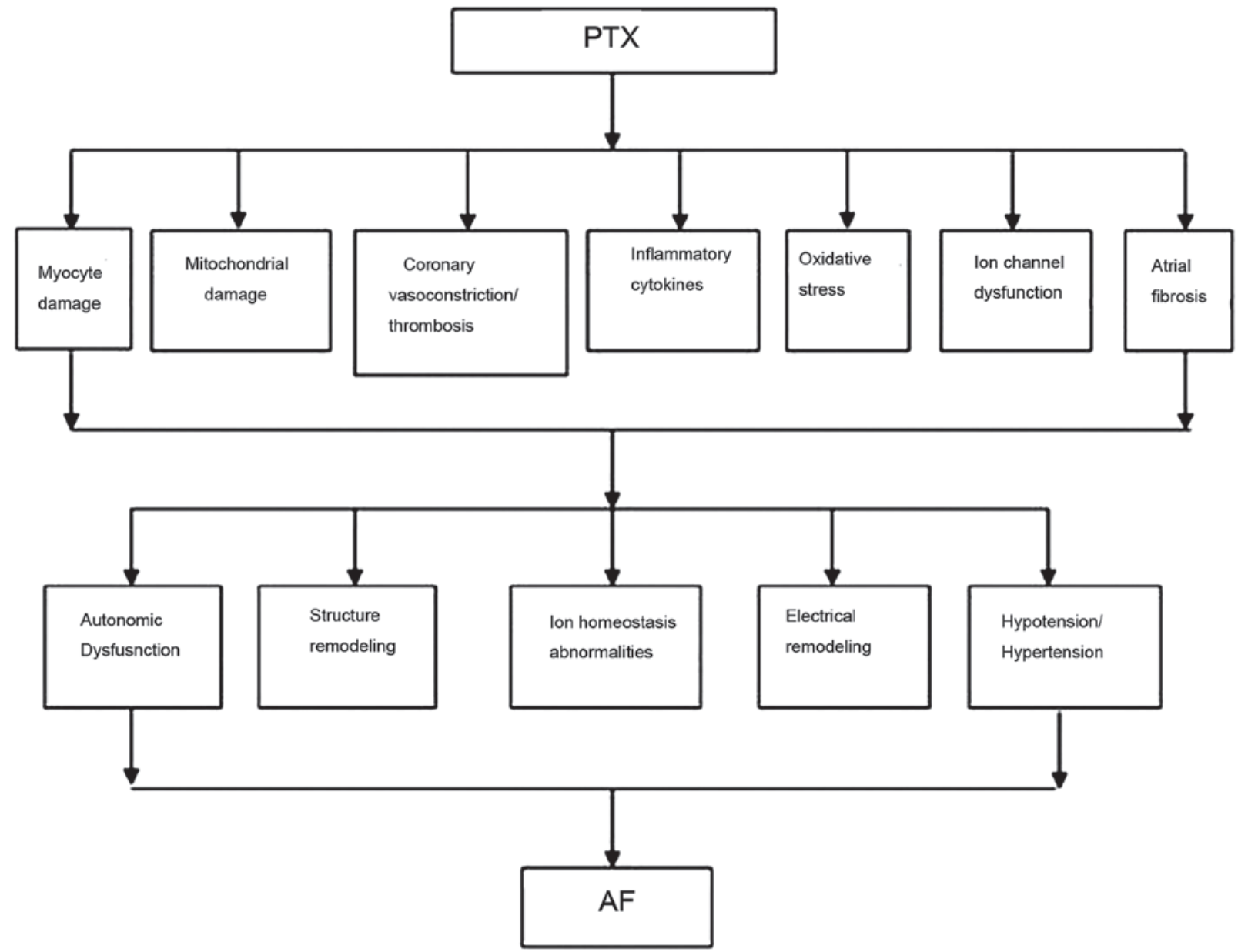

Figure 4. Schematic illustration of the mechanisms of atrial fibrillation (AF) induced by paclitaxel (PTX). 
addition to PTX, there were no other drugs that may cause AF, and therefore 2 points were given; and iv) the ECG indicated a rapid AF with rapid ventricular rate, and therefore 1 score point was given.

During the second chemotherapy cycle, except for mild nausea, no other adverse reactions were noted in the patient. Hematological and biochemical parameters prior to and following termination of the chemotherapy are listed in Table II. Except for hemoglobin (pre- and post-chemotherapy, 97 and $91 \mathrm{~g} / 1$, respectively; normal range 120-160 g/l), all of these parameters were normal. PTX was replaced with docetaxel in the next four chemotherapy cycles, and no AF occurred in the patient. However at 1 year on, the patient succumbed due to disease progression.

\section{Discussion}

$\mathrm{AF}$ is the most prevalent cardiac arrhythmia and a major cause of hospitalization, morbidity and mortality (5). PTX, an antimicrotubule agent, induces AF infrequently (10). In an analysis of $\sim 3,400$ cancer patients in the National Cancer Institute Adverse Drug Reactions database (13), atrial arrhythmias occurred in $>0.2 \%$ of patients who received PTX (5). Meanwhile, an increasing number of cardiovascular and non-cardiovascular drugs have been reported to induce AF (6). In the present case, the Naranjio algorithm was used to confirm the association between PTX and AF, which indicated that the occurrence of AF was likely to be associated with use of PTX.

The exact mechanism of AF induced by PTX is not well described or understood in the literature (10-12); however there are a number of hypotheses, including a stimulation of the sympathetic nervous system or parasympathetic nervous system to influence the function of the atrionector, or a stimulation of histamine 1 and 2 receptors to cause myocardial oxygen demand, coronary vasoconstriction and chronotropic effects $(5,6)$. A schematic flow chart of the potential mechanisms underlying PTX-induced AF is provided in Fig. 4. Considering the critical nature of this adverse reaction, attention should been paid when administering adjuvant chemotherapy with PTX, particularly in patients with possible or known cardiac disease (14).

AF typically lasts for a few minutes or hours in patients when the suspected drug is stopped (5). If AF persists for several hours or days following drug discontinuation $(5,6)$, the treatment is the same as that recommended for paroxysmal/persistent AF (5), with the common treatment procedures being drug withdrawal and control of rhythm and rate. However, the effectiveness of rhythm and rate control therapies on drug-induced AF has not been adequately studied, and guidelines are based exclusively on patients with cancer diseases (6). Certain studies $(5,6)$ have documented that amiodarone and $\beta 1$ blocker may be effective for rhythm and rate control. If the causative drug is necessary for the patient, the treatment can be started at a lower dose with continuous ECG monitoring to detect the recurrence of $\operatorname{AF}(5,6)$. Occasionally, it is possible to replace the causative agent for another drug of the same family (i.e., PTX replaced by docetaxel) (6).

In conclusion, AF is a rare, albeit critical adverse effect induced by PTX, and thorough observation should be performed during PTX treatment, even for patients with no previous presentation of cardiac risk factors. Further studies are required to establish the underlying mechanisms of PTX-induced AF in cancer patients, though preventive steps can be taken in the meantime.

\section{Acknowledgements}

The authors would like to thank Dr Shang Jingchuan (Department of Pharmaceutical Analysis, School of Pharmacy, Chongqing Medical University, Chongqing, China) for performing language editing of the manuscript.

\section{Funding}

No funding was received.

\section{Availability of data and materials}

All data described in the current report are available from the corresponding author on reasonable request.

\section{Authors' contributions}

DZ and XL were responsible for clinical evaluation and therapeutic management of the patient. DZ, JC and XL were responsible for the literature search. DZ, LC and JW were responsible for manuscript writing and provided corrections to the manuscript and figures. All authors reviewed and approved the final manuscript.

\section{Ethics approval and consent to participate}

Not applicable.

\section{Patient consent for publication}

The patient provided written consent for case publication.

\section{Competing interests}

The authors declare that they have no competing interests.

\section{References}

1. Cesca M, Morosi L, Berndt A, Fuso Nerini I, Frapolli R, Richter P, Decio A, Dirsch O, Micotti E, Giordano S, et al: Bevacizumabinduced inhibition of angiogenesis promotes a more homogeneous intratumoral distribution of paclitaxel, improving the antitumor response. Mol Cancer Ther 15: 125-135, 2016.

2. Brotto L, Brundage M, Hoskins P, Vergote I, Cervantes A, Casado HA, Poveda A, Eisenhauer E and Tu D; Gynecologic Cancer Intergroup Study of NCIC Clinical Trials Group (NCIC CTG); European Organization for Research and Treatment of Cancer - Gynecologic Cancer Group (EORTC-GCG); Grupo de Investigación de Cáncer de Ovario (GEICO): Randomized study of sequential cisplatin-topotecan/carboplatin-paclitaxel versus carboplatin-paclitaxel: Effects on quality of life. Support Care Cancer 24: 1241-1249, 2016.

3. Shah K, Gupta S, Ghosh J, Bajpai J and Maheshwari A: Acute non-ST elevation myocardial infarction following paclitaxel administration for ovarian carcinoma: A case report and review of literature. J Cancer Res Ther 8: 442-444, 2012.

4. Rawal G, Yadav S and Kumar R: Paclitaxel induced acute ST elevation myocardial infarction: A Rare Case Report. J Clin Diagn Res 10: XD01-XD02, 2016.

5. Kaakeh Y, Overholser BR, Lopshire JC and Tisdale JE: Drug-induced atrial fibrillation. Drugs 72: 1617-1630, 2012. 
6. Tamargo J, Caballero R and Delpón E: Drug-induced atrial fibrillation: Does it matter? Discov Med 14: 295-299, 2012.

7. Tsim S, O'Dowd CA, Milroy R and Davidson S: Staging of non-small cell lung cancer (NSCLC): A review. Respir Med 104: $1767-1774,2010$

8. Théophile H, André M, Miremont-Salamé G, Arimone Y and Bégaud B: Comparison of three methods (an updated logistic probabilistic method, the Naranjo and Liverpool algorithms) for the evaluation of routine pharmacovigilance case reports using consensual expert judgement as reference. Drug Saf 36 : 1033-1044, 2013

9. Belhekar MN, Taur SR and Munshi RP: A study of agreement between the Naranjo algorithm and WHO-UMC criteria for causality assessment of adverse drug reactions. Indian J Pharmacol 46: 117-120, 2014

10. Lombardi D, Crivellari D, Scuderi C, Magri MD, Spazzapan S, Sorio R, Di Lauro V, Scalone S and Veronesi A: Long-term, weekly one-hour infusion of paclitaxel in patients with metastatic breas cancer: A phase II monoinstitutional study. Tumori 90: 285-288, 2004.
11. Palma M, Mancuso A, Grifalchi F, Lugini A, Pizzardi N and Cortesi E: Atrial fibrillation during adjuvant chemotherapy with docetaxel: A case report. Tumori 88: 527-529, 2002.

12. Moscetti L, Ramponi S, Maccaglia C, Villanti P and Cortesi E: Atrial fibrillation in a patient with non-small-cell carcinoma of the lung in the course of paclitaxel therapy. Clin Ter 149: 377-379, 1998 (In Italian)

13. National Cancer Institute: National Cancer Institute Adverse Drug Reactions database. Available at: https://www.cancer.gov/.1993.

14. Zamorano JL, Lancellotti P, Muñoz DR, Aboyans V, Asteggiano R, Galderisi M, Habib G, Lenihan DJ, Lip GY, Lyon AR, et al: ESC Position Paper on cancer treatments and cardiovascular toxicity developed under the auspices of the ESC Committee for Practice Guidelines. Kardiol Pol 74: 1193-1233, 2016 (In Polish). 